\title{
Orientation specificity in chromatic adaptation of human "edge-detectors"1
}

\author{
L. S. FIDELL ${ }^{2}$ \\ THE UNIVERSITY OF MICHIGAN
}

The McCollough effect, a pattern-specific complementary hue aftereffect, has usually been demonstrated with horizontal and vertical adapting and test patterns. The present study measured the strength of the effect produced by adapting patterns of various angular separations. The effect decreased with decreasing angular separations until it was minimal at $11 \mathrm{deg}$ of separation. The results are considered to be consistent with an edge-detector interpretation of this aftereffect. With vertical and horizontal adapting patterns, the reddish aftereffect was $17 \%$ and the greenish aftereffect $9 \%$ of colorimetric purity.

McCollough (1965) produced two different aftereffects simultaneously by adapting is to vertical lines against an orange background alternated with horizontal lines against a blue background. A test stimulus composed of half vertical and half horizontal lines against a white background produced pattern-specific complementary hue aftereffects (i.e., the horizontal lines appeared orangish and the vertical lines blue-greenish). She attributed the effect to selective chromatic adaptation of two populations of edge-detectors (Hubel \& Wiesel, 1962) in human vision; the population of detectors for vertical lines had adapted to orange while the population of detectors for horizontal lines was adapting to blue.

Harris and Gibson (1968) have proposed another model for the McCollough effect based upon dipoles instead of edge-detectors. "The defining attribute of a dipole is that it receives inputs from two different locations on the retina. Like other sensory cells, dipoles are fatigued by prolonged stimulation, and the response will vary depending on what part of the spectrum a given dipole happens to be most sensitive to.... It is important to note only that lines of different orientations would tend to stimulate different populations of dipoles [Gibson \& Harris, 1968, pp. 9-10]."

Although offered as an alternative, the edge-detector hypothesis is not clearly different from the dipole hypothesis as currently defined. For example, areas of vertical excitation associated with areas of

Fig. 1. Adapting and patterned test stimuli used. vertical inhibition, or areas of concentric excitation and inhibition, also fit the definition of dipoles. Until each hypothesis is more stringently defined, experimental confirmation of only one of the two hypotheses is improbable. However, strong consistencies between physiological data on edge-detectors and psychophysical measures of the McCollough effect will lend credence to McCollough's original interpretation of the aftereffect.

Campbell et al (1968) and Hubel and Wiesel (1968) have determined the angular specificity of cortical cells selective to orientation in cat and monkey. Campbell's group found that the rate of firing of most orientation-specific neurons had fallen to

Angular

Condition Divergence

A

$90^{\circ}$ (90 vs 0$)$

$$
90^{\circ} \quad(135 \text { vs } 45)
$$
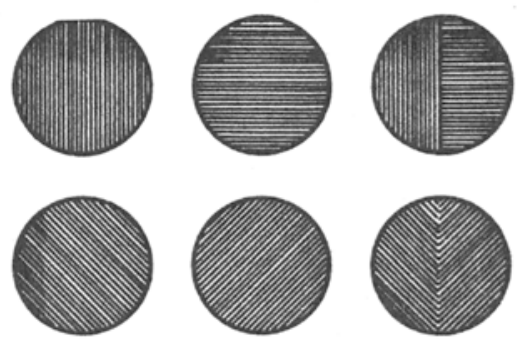

The present experiment determined the minimum angular divergence between

\section{Adapting Stimuli}

Patterned

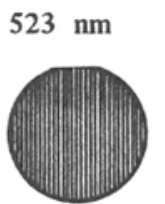

$650 \mathrm{~nm}$

Test Stimulus

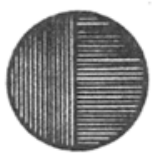

C

$90^{\circ}$ (158 vs 68)
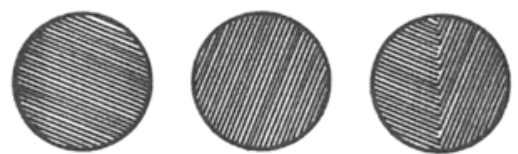

D

$90^{\circ}(169$ vs 79$)$
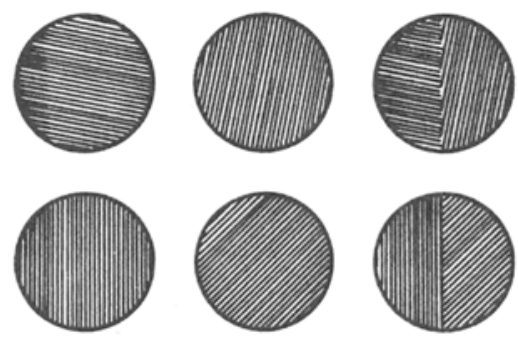

E

$45^{\circ}(90$ vs 45$)$
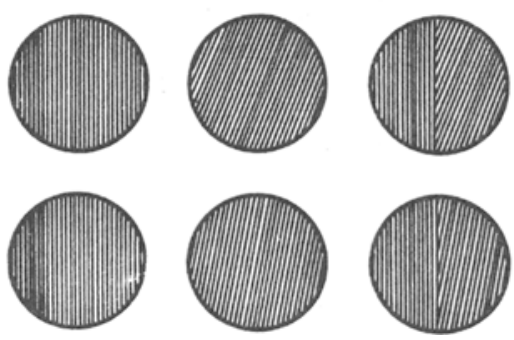
Fig. 2. Mean change in dial settings produced by adaptation in each condition. In Conditions $A$ to $D$ the adapting patterns were oriented at right angles to one another and no breakdown of the effect was observed (solid line). In Conditions E to $\mathrm{G}$ (broken line), in which the separation between adapting patterns progressively decreased, there was a progressive decrease in the aftereffect.

adapting patterns necessary to produce the McCollough effect and the intensity of the effect at different divergences. A before-after design was used in which responses gathered before adaptation were compared with those gathered after adaptation. Ss responded by giving verbal judgments of the hue of the test stimuli and then manually adjusting the hue of the test stimuli to neutral.

Thirty-five college-age paid Ss participated individually, five Ss in each of seven conditions. The Ss in Conditions A, B , C, and D saw two parallel-line adapting stimuli oriented at right angles to one another (although different pairs of orientations were used). In Conditions E, $F$, and $G$ Ss saw one adapting stimulus oriented vertically and the second adapting stimulus at 45,22 , or $11 \mathrm{deg}$ of angular divergence from vertical. Figure 1 displays adapting and patterned test stimuli employed in each condition.

Before testing began, Ss were instructed in experimental procedure, screened for color anomalies, and dark adapted for about $4 \mathrm{~min}$. Preadaptation testing ascertained Ss' biases for verbally reporting the presence of hues and for adjusting a stimulus to appear achromatic. Both kinds of responses were collected to an unpatterned control stimulus and to the right and left halves of the patterned test stimulus independently (cf. Fig. 1).

Verbal responses consisted of a hue-detection response ("hue" or "no hue") followed by a forced-choice hue-naming response ("blue," "green," "yellow," or "red").

The Ss also manually adjusted each stimulus to neutral before and after adaptation. Ss accomplished this by freely turning an unmarked dial which varied the colorimetric purity, but not the dominant wavelength or intensity of the test image projected to the screen. ${ }^{3}$ The final dial setting was recorded from a 14-point scale on which 1 represented selection of the achromatic beam, 14 represented selection of the chromatic beam, and intermediate numbers represented selection of varying proportions of the two beams.

The Ss adjusted the unpatterned control stimulus to neutral three times with red $(650 \mathrm{~nm})$ as the dominant wavelength and three times with green $(523 \mathrm{~nm})$ as the

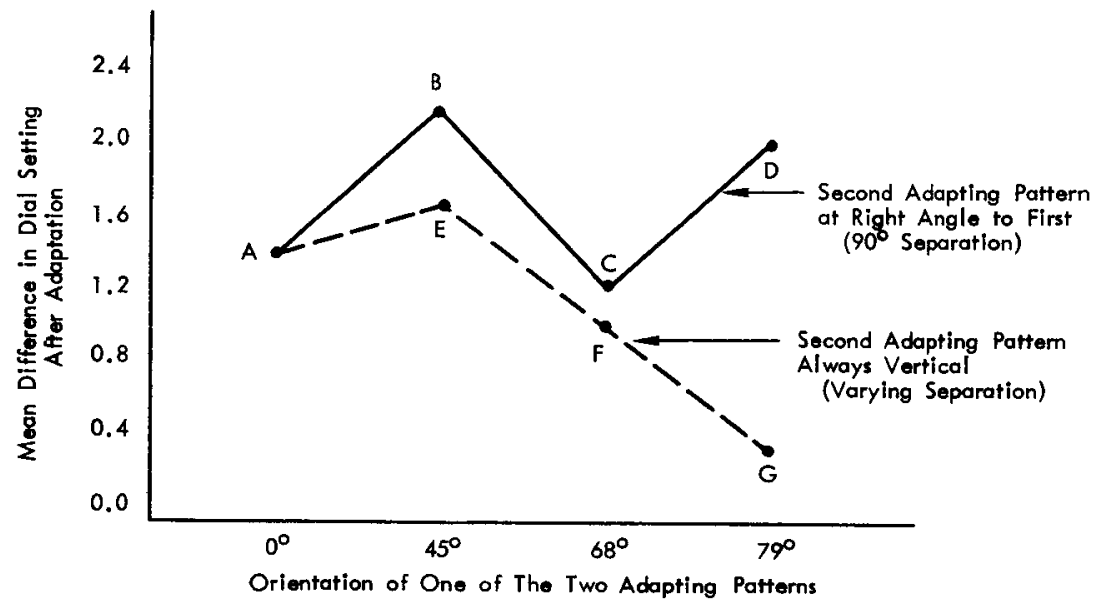

dominant wavelength both before and after adaptation. Each half of the patterned test stimulus was also adjusted to neutral before and after adaptation. With the patterned test stimulus, however, the dominant wavelength employed depended upon which color the pattern was paired with during adaptation; the pattern paired with red $(650 \mathrm{~nm})$ during adaptation was adjusted to neutral with red as the dominant wavelength (and similarly for green). If the McCollough effect was seen after adaptation, the half of the physically achromatic test stimulus which had been paired with red should appear greenish; the greenish aftereffect could then be neutralized by adding the appropriate amount of the original adapting hue to the test stimulus.

The difference in dial settings between pre- and postadaptation to the unupatterned control stimulus measured the (usually minimal) amount of generalized chromatic adaptation. The change in settings to the two halves of the patterned test stimulus measured the strength of the McCollough effect.

During adaptation, Ss sat with heads immobilized and observed the two adapting stimuli projected alternately to the screen every $5 \mathrm{sec}$ for $10 \mathrm{~min}$. Figure 1 shows which adapting pattern was paired with red and which was paired with green. The parallel lines of the stimuli were of equal black-transparent spacing such that 50 black lines appeared in the circular field (which subtended a visual angle of about $20 \mathrm{deg}$ ). The intensity of both the adapting and test stimuli was $0.03 \mathrm{~mL}$. The rest of the screen and the black-walled room were darkened.

After adaptation, Ss again judged the hue of the test stimuli and adjusted those stimuli to neutral. If "no hue" was reported after the initial $10 \mathrm{~min}$ of adaptation, adaptation was continued for an additional $5 \mathrm{~min}$. The same procedure was followed after a total of $15 \mathrm{~min}$ of adaptation. Twenty minutes of adaptation was an upper limit for all but one $S$ who received $30 \mathrm{~min}$ of adaptation.

\section{RESULTS}

The mean change in dial setting resulting from adaptation to adapting stimuli of the various angular divergences (for both sides of the test stimuli pooled together) is shown in Fig. 2. The breakdown of the effect at $11 \mathrm{deg}$ of angular divergence is quite apparent.

A Wilcoxon sign-rank analysis measured the consistency of direction and size of differences in dial settings resulting from adaptation. The differences in settings were significant for Ss in Conditions A (p less than 0.02 ) and $B, C$, and $D(p$ less than 0.01 ), the four conditions that maintained a 90-deg separation between adapting patterns. To render the test stimuli neutral, Ss added more hue to the test stimuli after adaptation than they had added before were found for Ss in Conditions $F$ and $G$ (22 and $11 \mathrm{deg}$ of angular separation), or for the unpatterned control stimulus.

The Ss reported "hue" much more frequently in Conditions $A, B, C$, and $D$. than in $E, F$, and $G$. Ninety percent of the responses were "hue" when there was a 90-deg separation between adapting patterns. At $45 \mathrm{deg}$ of separation, $40 \%$ were "hue" responses; at $22 \mathrm{deg}$ of separation there were $60 \%$ "hue" responses, while only $10 \%$ were "hue" responses with $11 \mathrm{deg}$ of separation between adapting patterns.

For Ss in Condition E, the discrepancy between the dial settings- which indicated that they experienced the aftereffect ( $p$ less than 0.01)-and the hue-detection responses-which were $60 \%$ "no hue"-may indicate that the adjustment procedure was more sensitive than verbal labeling in producing evidence of the effect.

Mean adaptation time to produce at least one "hue" response for all $\mathrm{Ss}$ in adaptation. No such consistent differences 
Conditions A to D was $10.5 \mathrm{~min}$. Mean adaptation times for Ss in Conditions $E, F$, and $G$ were 16,14 , and $20.5 \mathrm{~min}$, respectively. The prolonged periods of adaptation employed in the last three conditions were not always successful in producing the aftereffect.

\section{Measurement of the Strength of the Effect}

Since the aftereffect was produced readily with adapting stimuli at right angles, data generated by the patterned test stimuli of Conditions A to D were used to calculate the average strength of the McCollough effect under these adapting conditions. With green as the dominant wavelength, the mean preadaptation setting was 2.2 (standard error $=0.23$ ); the mean postadaptation setting was 4.2 (standard error $=0.30$ ). The difference of 2.0 units in mean setting may be interpreted as the strength of the reddish complementary hue aftereffect. Similarly, with red as the dominant wavelength, the mean preadaptation setting was 2.0 (standard error $=0.16)$, while the mean postadaptation setting was 3.4 (standard error $=0.14)$. The difference of 1.4 units was interpreted as the strength of the greenish aftereffect produced by viewing red.

Colorimetric purity values were obtained by linear interpolation of the proportions along the 14-point scale. With green as the dominant wavelength, $2 \%$ of colorimetric purity was considered neutral before adaptation; after adaptation, 19\% of colorimetric purity was necessary to cancel the reddish aftereffect. With red as the dominant wavelength, $3 \%$ of colorimetric purity was considered neutral before adaptation and $12 \%$ neutral after adaptation.

\section{DISCUSSION}

The consistency of the dial settings, the distribution of "hue" responses over conditions, and the mean times to successful adaptation all suggest that the strength of the McCollough effect is directly related to the angular separations of the adapting stimuli. Although aftereffects can apparently be produced to all orientations of adapting stimuli if they are at right angles to one another, aftereffects are not produced to patterns at the same orientations if the other adapting pattern is of less than 22-deg divergence.

The breakdown of the McCollough effect at $11 \mathrm{deg}$ of divergence between adapting patterns might have resulted from failure of both patterns to stimulate different populations of edge-detectors. This result is consistent with the physiological findings and supports an edge-detector explanation of the McCollough effect.

\section{REFERENCES}

CAMPBELL, F. W., CLELAND, B. G., COOPER, G. $F$., \& ENROTH-CUGELL, $C$. The angula selectivity of visual cortical cells to moving gratings. Journal of Physiology, 1968, 198, 237-250.

GIBSON, A. R., \& HARRIS, C. S. The McCollough effect: Color adaptation of edge-detectors or negative afterimages? Paper presented at the annual meeting of the Eastern Psychological Association, Washington, D.C., 1968.

HARRIS, C. S., \& GIBSON, A. R. Is orientation-specific color adaptation in human vision due to edge-detectors, after-images, or "dipoles"? Science, 1968, 162, 1506-1507.

HUBEL, D. H., \& WIESEL, T. N. Receptive fields, binocular interaction and functional architecture in the cat's visual cortex. Joumal of Physiology, 1962, 160, 106-154.

HUBEL, D. H., \& WIESEL, T. N. Receptive fieids and functional architecture of monkey striate cortex. Journal of Physiology, 1968, 195, 215-243.

McCOLLOUGH, C. Color adaptation of edge-detectors in the human visual system. Science, 1965, 149, 1115-1116.

\section{NOTES}

1. Research conducted as part of the requirements of the $\mathrm{PhD}$ degree at The University of Michigan and with support of funds from NIMH Fellowships 1-F 1-MH-29,060-01 and 5-F 1-MH-29,060-02 and a NSF summer traineeship.

2. Address. San Fernando Valley State College, Northridge, California 91364.

3. One half of a split beam of high intensity, achromatic light from a tungsten source was interrupted with a monochromatic interference filter of either dominant wavelength $523 \mathrm{~nm}$ or $650 \mathrm{~nm}$, the beams adjusted for intensity and remerged, the test image added, and the mix ture projected to the screen. The author will gladly supply more detail upon request.

(Accepted for publication January 7, 1970.) 